\title{
Modélisation numérique 2D unifiée des écoulements sur des évacuateurs de crue avec déversoir
}

\author{
Unified 2D numerical modelling of flows on ogee crest spillways
}

\section{BENJAMIN J. DEWALS*, SÉBASTIEN ERPICUM, PIERRE ARCHAMBEAU ET MICHEL PIROTTON}

Université de Liège, Département ArGEnCo, Unité d'Hydrologie, Hydrodynamique Appliquée et Constructions Hydrauliques, Institut de Mécanique et Génie civil - Chemin des Chevreuils 1, Bât. B52/3+1 - 4000 Liège, Belgique, e-mail : b.dewals@ulg.ac.be, s.erpicum@ulg.ac.be,michel.pirotton@ulg.ac.be

* Fonds de la Recherche Scientifique F.R.S.-FNRS

$L$ e système de modélisation WOLF 2D permet de combiner plusieurs sous-modèles hydrodynamiques différents au sein d'un même domaine de calcul. En particulier, pour la simulation des écoulements au voisinage des évacuateurs de surface des barrages, les trois sous-modèles suivants ont été interconnectés : (i) un modèle de type "eaux peu profondes » classique, (ii) un modèle adapté à la simulation du franchissement du seuil topographique brusque directement en amont de l'évacuateur de crue et (iii) un modèle représentant précisément l'effet de la courbure du fond à la crête de l'évacuateur, le long du coursier et au pied de celui-ci. Ce dernier modèle, basé sur un formalisme analytique en coordonnées curvilignes dans le plan vertical, tient compte explicitement non seulement de la pente du fond mais également de la courbure de celui-ci dans le plan vertical. En conséquence, le profil de vitesse sur la hauteur d'eau n'est pas uniforme et la distribution de pression, modifiée sous l'action des forces centrifuges, s'écarte d'un profil hydrostatique. Ce modèle mathématique étendu a été implémenté au sein du système de modélisation WOLF 2D, basé sur un schéma de discrétisation volumes finis inédit. Plusieurs exemples de validation et une application réelle démontrent la précision ainsi que l'applicabilité pratique du nouveau modèle.

$T$ he modelling system WOLF 2D enables to combine different hydrodynamic sub-models within one single computation domain. For simulating flows on ogee crest spillways, the following three depth-averaged submodels have been interconnected : (i) a conventional "shallow-water" model, (ii) a model suitable for the simulation of the flow on the topographic step directly upstream of the spillway and (iii) a model able to accurately account for the bottom curvature near the spillway crest, along the spillway and at its bottom. This third model, based on curvilinear coordinates in the vertical plane, takes into consideration not only the bottom slope but also the bottom curvature in the vertical plane. As a result, the velocity profile along the water depth is not uniform as assumed in the standard shallow-water equations. Similarly, centrifugal forces result in non-hydrostatic pressure distributions. This extended mathematical model has been implemented within the modelling system WOLF 2D, based on a new finite volume scheme. Several validation examples and a real application have demonstrated the accuracy and the practical applicability of the new model.

\section{INTRODUCTION}

Malgré les progrès permanents des outils de modélisation numérique, les études hydrauliques des organes d'évacuation de crue sont encore généralement réalisées par voie expérimentale. En effet, aucun logiciel de calcul ne peut se prévaloir aujourd'hui de représenter de manière suffisamment fiable et précise l'ensemble des phénomènes physiques qui régissent les écoulements au voisinage et sur les organes d'évacuation d'ouvrages de retenue (champs de vitesse tridimensionnels et complexes, turbulence, aération,...). En outre, pour autant que les lois de similitude adéquates soient res- pectées, les modèles physiques se distinguent par leur faculté encore inégalée à appréhender l'ensemble des spécificités de tels écoulements.

Les modèles numériques hydrodynamiques apportent tout de même nombre d'éléments de réponse pertinents lors de l'étude d'ouvrages même complexes. Ils se substituent valablement et efficacement à une partie des études sur modèle réduit, limitant ainsi l'ampleur des campagnes expérimentales nécessaires.

En particulier, le système de modélisation numérique WOLF, développé à l'Université de Liège, possède des fonctionnalités qui le rendent particulièrement adéquat pour 
simuler les écoulements à proximité et sur des évacuateurs de crue. Ces modèles hydrodynamiques ont été mis au point au cours de plusieurs thèses de doctorat (entre autres : [1-2]) et ont été validés au moyen de nombreuses données mesurées sur site ou acquises expérimentalement, notamment au Laboratoire de l'Université de Liège [3-8].

Ces modèles résolvent les « équations des eaux peu profondes » par une technique de type volumes finis. La discrétisation spatiale du domaine de calcul est effectuée au moyen de maillages réguliers multi-blocs $[1,6]$. Ceux-ci permettent non seulement d'adapter localement la finesse du maillage, mais aussi de combiner plusieurs sous-modèles hydrodynamiques dans différentes régions d'un même domaine de calcul. Pour la simulation des écoulements rencontrés à proximité des évacuateurs de surface des barrages, deux modèles additionnels ont été mis en œuvre pour compléter le modèle hydrodynamique de base (Modèle 0) :

- l'un pour reproduire l'écoulement au droit d'un dénivelé topographique brusque, comme généralement présent au niveau du seuil en amont de l'évacuateur de crue [1] (Modèle 1) ;

- l'autre pour représenter l'écoulement sur un fond caractérisé par une courbure forte dans le plan vertical, telle que présente à la crête de l'évacuateur et au pied de celui-ci [3] (Modèles 2 et 3).

Ce dernier modèle repose sur un formalisme analytique en coordonnées curvilignes dans le plan vertical, sur base duquel les équations locales sont intégrées selon l'épaisseur de la lame fluide (section III).

Par conséquent, cette organisation « multi-modèles » du système de modélisation numérique WOLF permet de simuler de manière unifiée les écoulements qui se développent en amont du barrage (dans la retenue), sur l'évacuateur (crête, coursier lisse, pied de l'évacuateur) ainsi qu'en aval de l'ouvrage, le tout au sein d'un domaine de calcul d'un seul tenant. Le système de modélisation WOLF inclut également des modèles de turbulence $[4,7]$ et de transport solide par charriage ou en suspension [9], ainsi que la résolution d'équations additionnelles de moment [2].

Les paragraphes qui suivent présentent successivement l'utilité et les principales caractéristiques des modèles mathématiques et numériques développés, en mettant l'accent sur la modélisation de la courbure du fond ; puis plusieurs exemples de validation sont détaillés. Finalement, on présente l'application des modèles à la simulation des écoulements sur un ouvrage réel.

\section{II — COMPLÉMENTARITÉ DES MODÈLES HYDRODYNAMIQUES UTILISÉS}

Afin de mettre en évidence l'utilité de combiner plusieurs modèles hydrodynamiques au sein d'une même modélisation, considérons l'exemple simple de l'écoulement sur un déversoir standard (Figure 1).

\section{II.1 TRAITEMENT DU SEUIL TOPOGRAPHIQUE}

La démarche la plus immédiate pour simuler l'écoulement sur le déversoir standard à l'aide d'un modèle intégré sur l'épaisseur de la lame fluide consiste à reproduire la géométrie du déversoir par le biais d'un obstacle topographique (Modèle 0). La hauteur calculée en amont par une telle approche vaut $0,88 \mathrm{~m}$, soit près de $9 \%$ de trop par rapport à la référence $(0,81 \mathrm{~m})$. Cet écart provient principalement du traitement numérique de la discontinuité topographique au niveau du seuil, de l'hypothèse de distribution hydrostatique de pression selon un axe vertical, sous-jacente au modèle classique des eaux peu profondes, ainsi que de l'hypothèse de vitesses faibles selon la direction verticale.

Par cette approche, l'impact sur l'écoulement du seuil topographique n'est donc pas reproduit de manière satisfaisante, d'autant que, dans cette formulation basée sur la conservation de la quantité de mouvement, le terme repré-

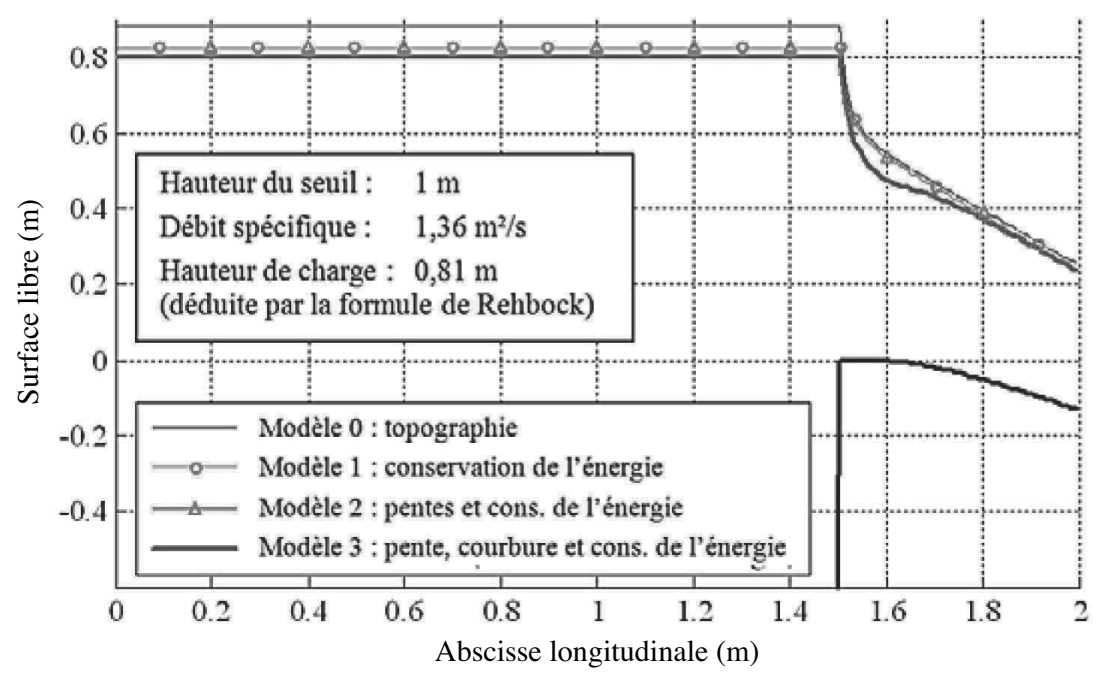

Figure 1 : Profil de surface libre calculé sur un déversoir standard, avec prise en compte de celui-ci par la topographie (avec et sans correction d'énergie) et par le calcul explicite de la pente et de la courbure du fond. 
sentatif de la pente de fond ne peut s'écrire sous une forme conservative, si bien que sa discrétisation numérique devient totalement imprécise en présence de variations brusques du fond [1].

La formulation alternative écrite en termes des inconnues primitives (hauteur et vitesses), traduisant la conservation de la charge, fait intervenir la pente de fond au sein d'un terme conservatif (Modèle 1). Valable pour autant que l'écoulement reste continu (i.e. en l'absence de ressaut), elle permet donc de calculer avec une précision accrue la ligne d'eau au droit du seuil topographique. La hauteur en amont se réduit alors à $0,825 \mathrm{~m}$ (Figure 1). Cette amélioration du résultat se révèle plus que substantielle, tout en ne dispensant pas de corriger également les autres sources de défaut de réalisme dans la modélisation.

\section{II.2 TRAITEMENT DE LA FORTE COURBURE DE L'ÉCOULEMENT DANS LE PLAN VERTICAL}

L'hypothèse d'une composante verticale de vitesse faible sous-tend le modèle classique des eaux peu profondes, avec comme conséquence immédiate une distribution hydrostatique de la pression selon la direction verticale. En réalité, la direction principale d'écoulement s'incline significativement dès l'aval de la crête du déversoir et, de façon concomitante, la distribution de pression s'écarte d'un profil hydrostatique selon la verticale. Il convient dès lors de définir localement une direction principale d'écoulement, de telle sorte que l'hypothèse sur la composante faible de vitesse, ainsi que son incidence sur l'orientation du profil de pression, soit à tout moment exprimée selon la direction la plus judicieuse. Formellement, cette opération est possible en établissant analytiquement les équations des eaux peu profondes dans un système d'axes cartésiens inclinés par rapport à l'horizontale [10-11], ce qui permet de réellement procéder à une intégration sur l'épaisseur locale de la lame fluide et non pas simplement selon la verticale. Ce faisant, bien que la Figure 1 montre que la hauteur en amont du déversoir standard n'est que très légèrement affectée $(0,823 \mathrm{~m})$, la ligne d'eau évolue dans le bon sens (Modèle 2).

Cette approche se heurte toutefois à une incohérence théorique. En effet, définir une valeur locale de l'inclinaison des axes revient à rendre les angles correspondants dépendants des coordonnées de l'espace. En conséquence, l'établissement des équations des eaux peu profondes doit être revu, afin de tenir compte du caractère variable de ces pentes, en prenant en considération non seulement leur valeur distribuée dans l'espace mais aussi leur taux de variation, i.e. la courbure $d u$ fond dans le plan vertical. Ainsi, une intégration analytique effectuée rigoureusement selon la direction localement normale à l'écoulement moyen conduit in fine au formalisme décrit à la section III, impliquant non seulement l'inclinaison locale des axes, mais aussi la courbure locale du fond. Comme en atteste la Figure 1, l'application de ce modèle généralisé permet une fois de plus d'accroître la précision du calcul (Modèle 3).

En résumé, cette généralisation du modèle des eaux peu profondes offre les avantages suivants pour décrire l'écoulement sur un fond présentant une courbure marquée dans le plan vertical : (i) l'hypothèse de vitesse faible est formulée selon une direction localement normale au fond et donc pas nécessairement selon la verticale ; (ii) la contribution hydrostatique au profil de pression est correctement orientée selon le même axe localement perpendiculaire au fond ; (iii) une correction non-hydrostatique à la distribution de pression est prise en compte pour traduire l'effet centrifuge induit par la courbure du fond.

\section{MODÈLE DES EAUX PEU PROFONDES GÉNÉRALISÉ}

La première prise en compte des effets de courbure verticale au sein d'un modèle d'écoulement intégré sur la hauteur d'eau a été réalisée par Dressler en 1978 [12], sans recourir à des équations additionnelles, mais en aboutissant à une formulation non conservative. Plusieurs validations expérimentales de ce premier modèle ont été exposées par Sivakumaran et al. [13-14]. Une généralisation bidimensionnelle du modèle, ainsi qu'une résolution de celui-ci par la méthode des éléments finis, ont été proposées par Berger et Carey [15-16]. La première implémentation du formalisme généralisé conservatif au sein d'un modèle de type volumes finis a été présentée par Dewals et al. [3]. Récemment, Anh et Hosoda [17] ont dérivé une formulation du modèle des écoulements peu profonds sur une surface courbe arbitraire, tandis que Castro-Orzag et Dey [18] ont modifié en 1D le modèle de Dewals et al. [3] en y introduisant les tensions turbulentes. En parallèle, plusieurs modèles 3D ont également été appliqués à la simulation d'écoulements sur les évacuateurs de crues (ex. [19]), tandis que d'autres auteurs ont modélisé en deux dimensions des écoulements sur fond à forte courbure par le biais d'équations de moment[20-21]. Cependant, cette dernière approche présente l'inconvénient de faire intervenir dix équations en $2 \mathrm{D}$, alors que l'approche privilégiée ici ne nécessite la résolution que de trois équations.

La dérivation complète du modèle à partir d'un repère en coordonnées curvilignes ( $\xi, y, \eta$, voir Figure 2) dans le plan vertical est détaillée dans Dewals et al. [3]. Elle conduit in fine au formalisme suivant :

$$
\begin{aligned}
\frac{\partial \mathcal{H}}{\partial t}+\frac{\partial}{\partial \xi}\left[H C \frac{\ln (1-\kappa H)}{-\kappa H}\right]+\frac{\partial}{\partial y}(\mathcal{H} V)=0 \\
\text { avec } \mathcal{H}=H\left(1-\frac{\kappa H}{2}\right) \text { et } \kappa H<1,
\end{aligned}
$$

$$
\begin{aligned}
& \frac{\partial}{\partial t}(H C)+\frac{\partial}{\partial \xi}\left(\frac{H C^{2}}{1-\kappa H}\right)+\frac{\partial}{\partial y}(H C V) \\
& +\frac{\partial}{\partial \xi}\left[g \frac{H^{2}}{2} \cos \theta+\frac{H C^{2}}{2} \frac{\kappa H}{(1-\kappa H)^{2}}\right] \\
& \quad=-g \mathcal{H} \sin \theta+\kappa H \int_{0}^{H}(1-\kappa \eta)^{-1} C W d \eta
\end{aligned}
$$




$$
\begin{array}{r}
\frac{\partial}{\partial t}(\mathcal{H} V)+\frac{\partial}{\partial \xi}\left[V H C \frac{\ln (1-\kappa H)}{-\kappa H}\right]+\frac{\partial}{\partial y}\left(\mathcal{H} V^{2}+g \frac{H^{2}}{2} \cos \theta\left(1-\frac{\kappa H}{3}\right)\right. \\
\left.+\frac{H C^{2}}{2} \frac{1-\kappa H / 2}{(1-\kappa H)^{2}}-\frac{H C^{2}}{2} \frac{\ln (1-\kappa H)}{-\kappa H}\right)=0
\end{array}
$$

avec $H=$ hauteur d'eau mesurée selon $\eta, \kappa=$ courbure du fond, $C=$ composante selon $\xi$ de la vitesse d'écoulement sur le fond, $V=$ composante selon $y$ de la vitesse moyenne, $\theta=$ angle local entre le fond et l'horizontale, $t=$ temps, $W=$ composante selon $\eta$ de la vitesse d'écoulement.
Le système d'équations obtenu reste conservatif comme le modèle classique, ce qui constitue un atout essentiel pour la discrétisation numérique. En l'occurrence, une approche volumes finis est utilisée pour la résolution numérique des différents modèles. Elle repose notamment sur un schéma de discrétisation original de type " fractionnement des flux » (fonction du signe de la courbure du fond), dont la stabilité numérique a été démontrée théoriquement par une analyse de von Neumann [2] puis vérifiée en pratique. L'architecture multiblocs du système de modélisation [1] permet de concentrer la résolution de ce modèle généralisé dans les zones où il se justifie (ex. crête d'un déversoir), tandis que le modèle classique ou celui basé sur la conservation de l'énergie sont résolus ailleurs.
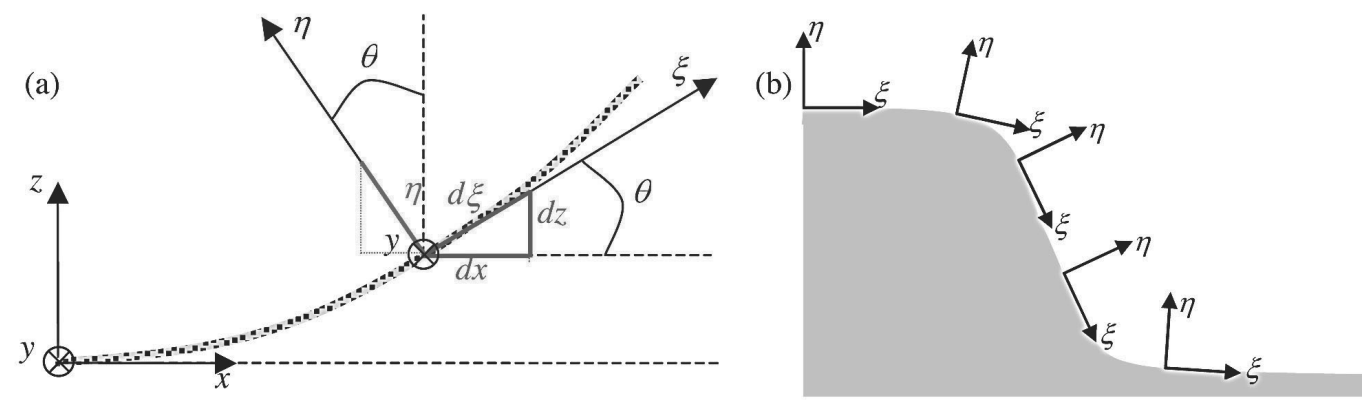

Figure 2 : (a) Axes cartésiens $(x, y, z)$ et curvilignes $(\xi, y, \eta)$. (b) Exemple d'inclinaison des axes le long d'un déversoir.

\section{VALIDATION}

Un premier exemple de validation concerne l'écoulement dans un canal vitré comportant un obstacle symétrique, tel qu'étudié expérimentalement par Sivakumaran et al. [13-14]. La Figure 3(a) montre la surestimation notoire de la hauteur d'eau en amont de l'obstacle par une modélisation ne tenant pas explicitement compte de la courbure du fond (erreur de 7 \%). En revanche, comme le montre la Figure 3(b), les hauteurs d'eau calculées par le modèle généralisé se rapprochent très nettement des résultats expérimentaux (erreur inférieure à $1 \%$ ), tandis que le profil de pression est aussi nettement mieux reproduit.

Un second exemple de validation porte sur la modélisation de l'écoulement sur un déversoir terminé par une cuillère [21]. La simulation appréhende d'un seul tenant l'écoulement en amont du déversoir, sur la crête, sur le coursier ainsi que sur la cuillère. Les hauteurs d'eau sont valablement appro-
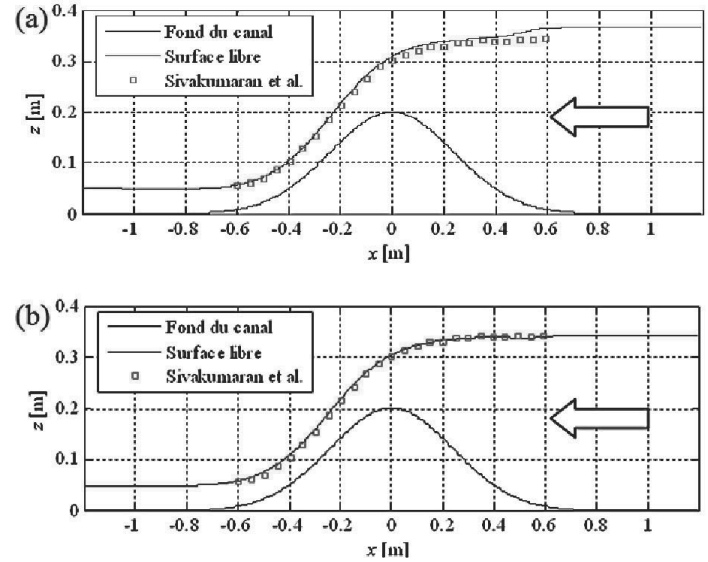

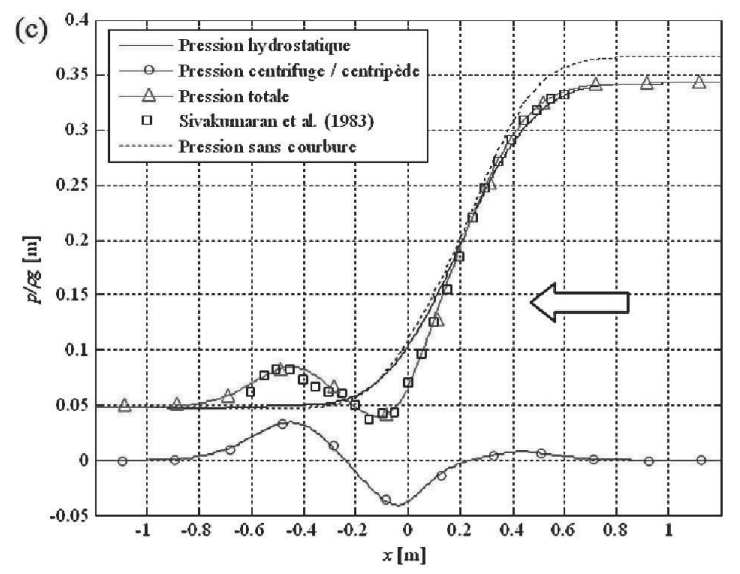

Figure 3 : Modélisation de l'écoulement sur un profil symétrique $\left(n=0,01 \mathrm{~s} / \mathrm{m}^{1 / 3}, q=0,11197 \mathrm{~m}^{2} / \mathrm{s}\right)$ sans prise en compte de la courbure du fond (a) et avec prise en compte explicite de celle-ci (b). Profils de pression obtenus (c). 

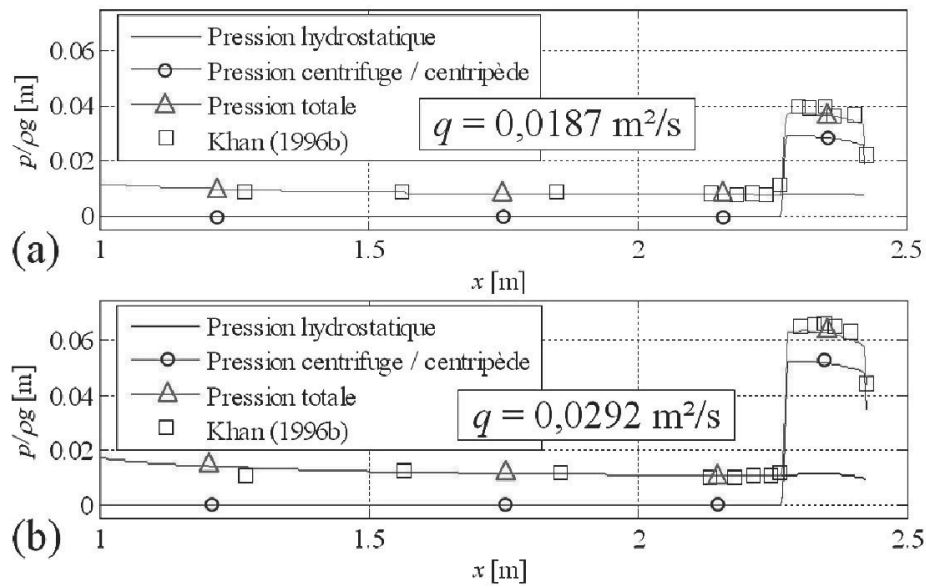

Figure 4 : Pression le long d'un déversoir terminé par une cuillère pour deux débits spécifiques.

chées tant par le modèle classique (Modèle 0) que par la formulation tenant compte explicitement de la courbure, ce qui découle notamment de la valeur très réduite du nombre de Froude en amont. En revanche, la comparaison des valeurs simulées et mesurées de la pression sur le fond concave de la cuillère (Figure 4), où les effets centrifuges sont particulièrement marqués, met en évidence la remarquable précision du modèle généralisé en ce qui concerne la surpression au niveau de la cuillère, alors que cet accroissement local de pression est gommé par le modèle classique. Les données expérimentales proviennent des essais de Khan et Steffler [21].

\section{D APPLICATION}

Edifié en 1958, le barrage de Nisramont se situe sur le cours supérieur de l'Ourthe, un des principaux affluents de la Meuse en Belgique. Haut de $21 \mathrm{~m}$ et disposant d'un bassin versant de $740 \mathrm{~km}^{2}$, l'ouvrage retient un réservoir de $3 \mathrm{hm}^{3}$, qui sert à approvisionner en eau potable et industrielle une grande partie du sud de la Belgique. Lors de sa construction, le barrage était pressenti comme batardeau provisoire en vue de l'édification d'un autre ouvrage de retenue de plus grande ampleur en aval. Ce dernier n'ayant jamais vu le jour, le barrage de Nisramont est devenu définitif.
Le barrage est équipé d'un évacuateur de surface constitué de trois pertuis de 12,5 $\mathrm{m}$ de large chacun (débit maximum : $427 \mathrm{~m}^{3} / \mathrm{s}$ ), ainsi que de trois vannes de fond (Figure 5). En raison de la vocation initialement temporaire du barrage et suite aux révisions récentes des statistiques hydrologiques, il convenait de procéder à une vérification du dimensionnement du bassin de dissipation situé en aval de l'ouvrage. C'est pourquoi, les modèles hydrodynamiques décrits précédemment ont été exploités pour contribuer au redimensionnement de ce bassin de dissipation, non sans avoir été au préalable calibrés et vérifiés par comparaison avec des observations effectuées lors d'une crue récente sur le site du barrage [22]. En effet, le passage sur le barrage d'un débit de $175 \mathrm{~m}^{3} / \mathrm{s}$ le 3 janvier 2003 a été documenté sur base de nombreuses photos, fournissant des informations quantitatives utiles pour la validation du modèle.

Comme le détaille la Figure 6, le domaine de simulation a été découpé en quatre blocs discrétisés au moyen de mailles carrées de $25 \mathrm{~cm}$ à $1 \mathrm{~m}$ de côté. Un bloc a été défini en amont de l'ouvrage (Bloc 1), un sur le coursier (Bloc 2), un troisième couvre la zone du bassin de dissipation (Bloc 3 ) et le dernier représente la rivière naturelle jusqu'à la limite aval du domaine (Bloc 4). La formulation classique des équations des eaux peu profondes (Modèle 0) est résolue dans les blocs 3 et 4 , tandis que, dans le bloc 2, le modèle tenant

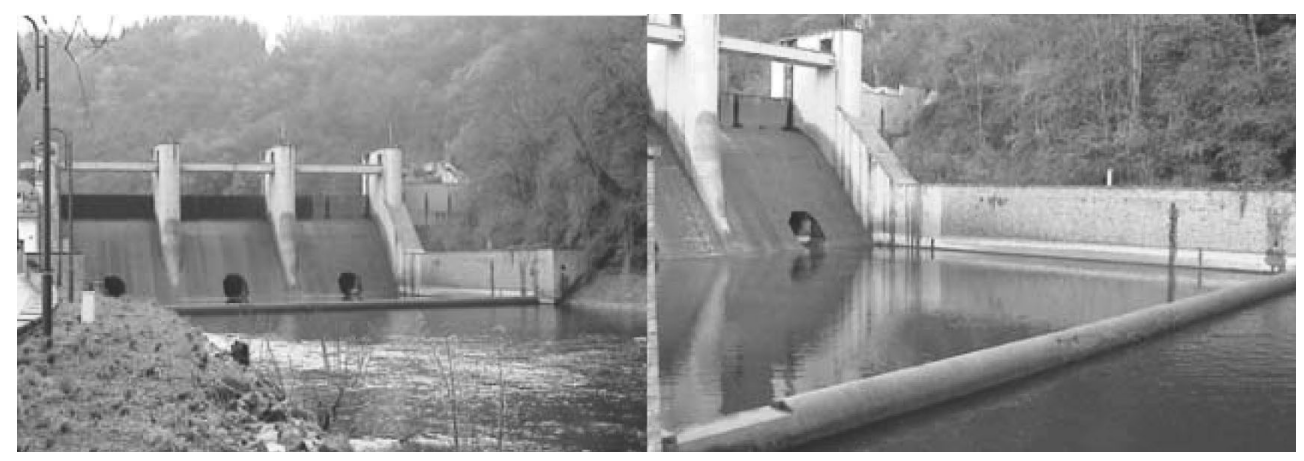

Figure 5 : Barrage poids en béton de Nisramont et détail du coursier ainsi que du bassin de dissipation 
Bloc 2 (Déversoir - modèle coordonnées curvilignes)

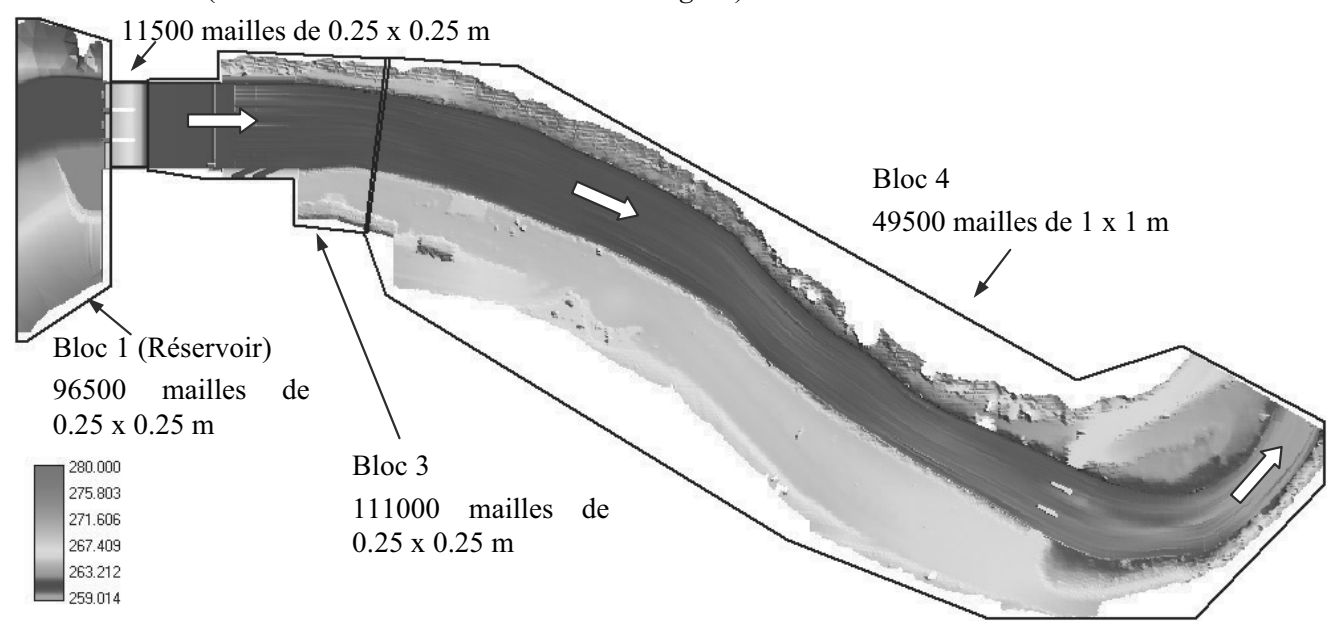

Figure 6 : Topographie du domaine de modélisation et définition des différentes régions du domaine pour l'approche multi-blocs et multi-modèles.

compte de la forte courbure du fond dans le plan vertical est exploité et, dans le bloc 1, il s'agit du modèle de type " conservation de l'énergie » (Modèle 1) qui a été retenu de sorte que la charge en amont de l'ouvrage, et en particulier en amont du seuil, soit correctement évaluée.

Les seules conditions limites imposées au modèle étaient l'altitude de surface libre dans la rivière 600 mètres en aval de l'ouvrage, ainsi que la répartition en plan du débit dans la retenue en amont du barrage, répartition issue d'une simulation globale préalable des écoulements dans l'ensemble du réservoir. C'est ici qu'apparaît clairement l'originalité et l'intérêt d'une modélisation unifiée amont-aval, par exemple pour le calcul objectif de la courbe charge-débit caractéristique d'un tel ouvrage.

Une étude de sensibilité portant sur le coefficient de frottement des structures dans sa gamme de variation physiquement réaliste a certes montré la sensibilité de la position du ressaut dans le bassin de dissipation par rapport à ce paramètre, mais l'analyse a également démontré l'impossibilité de positionner correctement le ressaut dans le bassin de dissi- pation sans utiliser la forme des équations des eaux peu profondes tenant explicitement compte de la courbure du fond. Les valeurs du coefficient de frottement de Manning égale à $0,014 \mathrm{~s} / \mathrm{m}^{1 / 3}$ pour les parties bétonnées de l'ouvrage et égale à $0,037 \mathrm{~s} / \mathrm{m}^{1 / 3}$ pour le lit naturel de la rivière conduisent au meilleur accord avec les observations en ce qui concerne la position du ressaut dans le bassin de dissipation, les hauteurs d'eau dans la rivière en aval notamment au niveau des berges, ainsi que la position et la géométrie des cross-waves et des zones d'assèchement au pied des piles (Figure 7).

\section{CONCLUSION}

Le système de modélisation présenté offre l'avantage de permettre la combinaison de plusieurs modèles hydrodynamiques 2D distincts au sein d'un même domaine de simulation. Parmi ces modèles, outre l'approche classique des eaux peu profondes, formulée soit en termes de conservation de la quantité de mouvement soit en termes de conservation
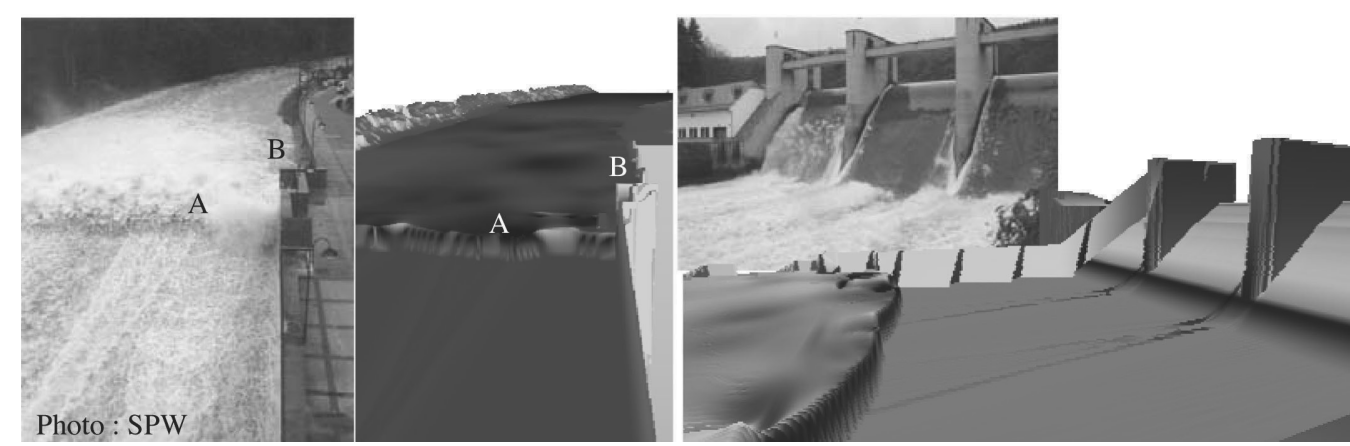

Figure 7 : Simulation de la crue du 3 janvier 2003 - Débit $=175 \mathrm{~m}^{3} / \mathrm{s}$ - Validation de l'approche numérique : comparaison de l'événement réel et des résultats simulés. 
de l'énergie, figure également une généralisation du modèle des eaux peu profondes tenant compte explicitement de la courbure du fond au moyen d'un formalisme analytique en coordonnées curvilignes dans le plan vertical. Cette nouvelle formulation, considérant des profils enrichis des champs de vitesse et de pression sur la hauteur d'eau a été implémentée au sein d'un code "volumes finis » et un schéma de discrétisation spécifique de type « fractionnement des flux » a été élaboré. La comparaison des résultats simulés avec des mesures expérimentales met en exergue les apports significatifs du modèle généralisé par rapport au modèle classique, tant en termes de précision du profil de hauteurs d'eau que de distribution de pression sur le fond. Une telle approche généralisée présente donc l'avantage d'étendre les possibilités de modélisation intégrée sur l'épaisseur de la lame fluide, permettant ainsi d'éviter dans certains cas le recours à une discrétisation 3D nécessairement plus coûteuse en temps de calcul. Plus globalement, la capacité du système de modélisation à traiter de façon unifiée l'écoulement en amont d'un ouvrage de retenue, sur celui-ci et en son aval, ouvre la porte à de vastes possibilités d'applications numériques, parmi lesquelles le dimensionnement d'ouvrages, la quantification des risques d'alluvionnement en amont, ou encore la prédiction des fosses d'affouillement en aval, ...

\section{VII —RÉFÉRENCES}

[1] ERPICUM S. (2006) - Optimisation objective de paramètres en écoulements turbulents à surface libre sur maillage multibloc. Thèse de doctorat, Université de Liège. $356 \mathrm{p}$

[2] Dewals B. (2006) - Une approche unifiée pour la modélisation d'écoulements à surface libre, de leur effet érosif sur une structure et de leur interaction avec divers constituants. Thèse de doctorat, Université de Liège. $636 \mathrm{p}$

[3] Dewals B. J., Erpicum S., Archambeau P., Detrembleur S., Pirotton M. (2006) - Depth-integrated flow modelling taking into account bottom curvature. J. Hydraul. Res. 44(6) 787-795

[4] Dewals B. J., Kantoush S. A., Erpicum S., Pirotton M., SCHLEISS A. J. (2008) - Experimental and numerical analysis of flow instabilities in rectangular shallow basins. Environ. Fluid Mech. 8 31-54

[5] Erpicum S., Dewals B. J., Archambeau P., Detrembleur S., Pirotton M. (2010) - Detailed inundation modelling using high resolution DEMs. Engineering Applications of Computational Fluid Mechanics. 4(2) 196-208

[6] Erpicum S., Dewals B. J., Archambeau P., Pirotton M. (2009) - Dam-break flow computation based on an efficient flux-vector splitting. J. Comput. Appl. Math. 234 2143-2151

[7] Erpicum S., Meile T., Dewals B. J., Pirotton M., Schleiss A. J. (2009) $-2 \mathrm{D}$ numerical flow modeling in a macro- rough channel. Int. J. Numer. Methods Fluids. 61(11) 12271246

[8] Roger S., Dewals B. J., Erpicum S., Pirotton M., Schwanenberg D., SchüttrumpF H., Köngeter J. (2009) - Experimental und numerical investigations of dike-break induced flows. J. Hydraul. Res. 47(3) 349-359

[9] Dewals B. J., Erpicum S., Archambeau P., Detrembleur S., Pirotton M. (2008) - Hétérogénéité des échelles spatiotemporelles d'écoulements hydrosédimentaires et modélisation numérique. La Houille Blanche. 5 109-114

[10] Andre S., Dewals B. J., Pirotton M., Schleiss A. (2003) - Quasi 2D-numerical model of aerated flow over stepped chutes, in Proc. 30th IAHR Congress, J. Ganoulis \& P. Prinos, Eds. IAHR : Thessalonique, Grèce. 671-678

[11] Dewals B. J., Andre S., Schleiss A., Pirotton M. (2004) Validation of a quasi-2D model for aerated flows over stepped spillways for mild and steep slopes. Proc. 6th Int. Conf. of Hydroinformatics. Singapore.

[12] Dressler R. F. (1978) - New Nonlinear Shallow Flow Equations with Curvature. J. Hydraul. Res. 16(3) 205-222

[13] Sivakumaran N. S., Hosking R. J., Tingsanchali T. (1981) - Steady shallow flow over a spillway. J. Fluid Mech. 111 $411-420$

[14] Sivakumaran N. S., Tingsanchali T., Hosking R. J. (1983) - Steady shallow flow over curved beds. J. Fluid Mech. 128 469-487

[15] Berger R. C., Carey G. F. (1998) - Free-surface flow over curved surfaces - Part I : perturbation analysis. Int. J. Numer. Meth. Fluids. 28(2) 191-200

[16] Berger R. C., Carey G. F. (1998) - Free-surface flow over curved surfaces - Part II : computational model. Int. J. Numer. Meth. Fluids. 28(2) 201-213

[17] Anh T. N., Hosoda T. (2007) - Depth-averaged model of open-channel flows over an arbitrary 3D surface and its applications to analysis of water surface profile. J. Hydraul. Eng.ASCE. 133(4) 350-360

[18] Castro-Orzag O., Dey S. (2008) - One-dimensional channel flow equations with curvature revisited. J. Hydraul. Res. 47(2) $157-166$

[19] BÜrgisser M. F., Rutschmann P. (1999) - Numerical solution of viscous $2 \mathrm{DV}$ free surface flows : flow over spillway crests. 28th IAHR Congress Graz. Graz, Austria.

[20] Khan A. A., Steffler P. M. (1996) - Modelling overfalls using vertically averaged and moment equations. J. Hydraul. Eng.-ASCE. 122(7) 397-402

[21] Khan A. A., Steffler P. M. (1996) - Vertically averaged and moment equations model for flow over curved beds. $J$. Hydraul. Eng.-ASCE. 122(1) 3-9

[22] Erpicum S., Archambeau P., Dewals B., Detrembleur S., Pirotton M. (2006) - Decision making numerical tools for the rehabilitation of hydraulic structures - Application to the Nisramont dam in Belgium. ICOLD Congress. Barcelone, Espagne 